\title{
TRANSFORMATIONS OF APPLICABLE CONJUGATE NETS OF CURVES ON SURFACES*
}

BY

\section{LUTHER PFAHLER EISENHART}

In a previous paper $\dagger$ we developed the theory of transformations $T$ of conjugate systems of curves on a surface into similar systems on other surfaces. In the present paper we are concerned with the application of these results to a particular class of conjugate systems, namely those which are applicable to one or more other systems. Thus if $S$ and $\bar{S}$ are two surfaces upon which the corresponding conjugate system is parametric, we say that the parametric nets are applicable when corresponding first fundamental coefficients are equal. In order to give this definition analytic form, we suppose that the cartesian coördinates of $S$ and $\bar{S}$ are $x, y, z$ and $\bar{x}, \bar{y}, \bar{z}$ respectively. Since we are interested particularly in parametric nets, we designate them by $N(x)$ and $\bar{N}(\bar{x})$, or merely by $N$ and $\bar{N}$ whenever it is not necessary to specify the coördinates. The first fundamental coefficients are defined by

$$
\begin{array}{lll}
E=\sum\left(\frac{\partial x}{\partial u}\right)^{2}, & F=\sum \frac{\partial x}{\partial u} \frac{\partial x}{\partial v}, & G=\sum\left(\frac{\partial x}{\partial v}\right)^{2}, \\
\bar{E}=\sum\left(\frac{\partial \bar{x}}{\partial u}\right)^{2}, & \bar{F}=\sum \frac{\partial \bar{x}}{\partial u} \frac{\partial \bar{x}}{\partial v}, & \bar{G}=\sum\left(\frac{\partial \bar{x}}{\partial v}\right)^{2},
\end{array}
$$

the symbol $\sum$ indicating throughout the paper the sum of three terms obtained from the three coördinates. Hence the condition for the applicability of the nets $N$ and $\bar{N}$ is

$$
\begin{gathered}
\sum\left(\frac{\partial x}{\partial u}\right)^{2}=\sum\left(\frac{\partial \bar{x}}{\partial u}\right)^{2}, \quad \sum \frac{\partial x}{\partial u} \frac{\partial x}{\partial v}=\sum \frac{\partial \bar{x}}{\partial u} \frac{\partial \bar{x}}{\partial v}, \\
\sum\left(\frac{\partial x}{\partial v}\right)^{2}=\sum\left(\frac{\partial \bar{x}}{\partial v}\right)^{2} .
\end{gathered}
$$

The coördinates of $N$ satisfy an equation of the form

$$
\frac{\partial^{2} \theta}{\partial u \partial v}=a \frac{\partial \theta}{\partial u}+b \frac{\partial \theta}{\partial v}
$$

* Presented to the Society, February 23, 1918.

† These Transactions, vol. 18 (1917), pp. 97-124. 
where $a$ and $b$ are functions of $E, F, G$ and their derivatives. Consequently the coördinates of $\bar{N}$ satisfy the same equation. We call it the point equation of the nets.

Any net $N^{\prime}\left(x^{\prime}\right)$ whose tangents are parallel to the tangents to $N(x)$ at the point with the same parametric coördinates $(u, v)$ is said to be parallel to $N$. Peterson* showed that if $N$ and $\bar{N}$ are applicable nets, the knowledge of a net parallel to either leads by quadratures to the determination of a net parallel to the other, and the new nets $N^{\prime}$ and $\bar{N}^{\prime}$ are applicable. We extend this result by showing that each pair of nets parallel to $N$ and $\bar{N}$ leads by quadratures to the determination of another pair of applicable nets $N_{1}$ and $\bar{N}_{1}$ which are Ttransforms of $N$ and $\bar{N}$ respectively. We recall that in a transformation $T$ of a net $N$ the coördinates of a certain parallel net $N^{\prime}$ are the direction-parameters of the line joining corresponding points on $N$ and the transform $N_{1}$, and the further determination of $N_{1}$ requires a solution of equation (3). For the transformations now under discussion the corresponding solution of equation (3) is given by a quadrature after a parallel net is known.

Nets are of three types with regard to applicability. Nets of the first type do not admit any applicable nets. Those of the second type admit one applicable net each, whereas a net of the third type admits an infinity of applicable nets. We say that the latter are permanent in deformation, and for the sake of brevity call them permanent nets. Every net parallel to a permanent net is a permanent net, and each of the infinity of nets applicable to the one is parallel to one of the infinity applicable to the other by the method of Peterson. Suppose now that we have a permanent net $N$, two applicable nets $\bar{N}$ and $\bar{N}$, and the respective parallel and applicable nets $N^{\prime}, \bar{N}^{\prime}, \bar{N}^{\prime}$. By the process described in the preceding paragraph we obtain two transforms $N_{1}$ and $N_{2}$ of $N$, in general distinct, such that the corresponding points of $N, N_{1}, N_{2}$ lie on the same line, whose direction-parameters are the coördinates of $N^{\prime}$. At the same time we obtain two transforms of $\bar{N}$ and two of $\bar{N}$. As $N$ admits an infinity of applicable nets, this process can be extended with the result that in general $N$ and each of its transforms admit an infinity of transforms. We raise the question whether in any case this infinity of transforms are coincident for each of the nets, so that we obtain a permanent net $N_{1}$, whose infinity of applicable nets are the $T$ transforms of the nets applicable to $N$. We refer to this question as Problem $A$.

Permanent nets belong to the general class of nets whose tangential coördinates satisfy an equation of Laplace with equal invariants. We have established $\dagger$ transformations of nets of this kind into nets of the same kind. When

* Ueber Curven und Flachen, Moskau and Leipzig, 1868, p. 106. Also Stäckel, M a t h e matische Annalen, vol. 49 (1897), p. 255.

$\dagger$ R endiconti di Palermo, vol. 39 (1915), pp. 153-176. 
in particular $N$ is a permanent net, we find transformations furnishing a solution of Problem $A$.

In the transformations just referred to we did not consider permanent nets for which the curves in one family are represented on the Gauss sphere by one system of its imaginary generators. Drach* solved the problem of the deformation of nets of this kind. We show how in two ways these nets can be transformed into nets of the same kind as a solution of Problem $A$.

The third type of permanent nets are those whose two families of curves are represented on the Gauss sphere by the imaginary generators. These curves are the minimal curves on a minimal surface. There are no transformations of nets of this kind into similar nets furnishing a solution of Problem $A$.

\section{General transformations of apPlicable nets}

If $N(x)$ is a net whose cartesian coördinates satisfy equation (3), a parallel net $N^{\prime}\left(x^{\prime}\right)$ is defined by

$$
\frac{\partial x^{\prime}}{\partial u}=h \frac{\partial x}{\partial u}, \quad \frac{\partial x^{\prime}}{\partial v}=l \frac{\partial x}{\partial v},
$$

where $h$ and $l$ are a pair of solutions of

$$
\frac{\partial h}{\partial v}=(l-h) a, \quad \frac{\partial l}{\partial u}=(h-l) b .
$$

If $\theta$ is any solution of (3) and $\theta^{\prime}$ is the solution of the point equation of $N^{\prime}$ given by the quadrature

$$
\frac{\partial \theta^{\prime}}{\partial u}=h \frac{\partial \theta}{\partial u}, \quad \frac{\partial \theta^{\prime}}{\partial v}=l \frac{\partial \theta}{\partial v},
$$

then equations of the form

$$
x_{1}=x-\frac{\theta}{\theta^{\prime}} x^{\prime}
$$

define a net $N_{1}$ in the relation of a transformation $T$ with $N$, that is the developables of the congruence of lines joining corresponding points on $N$ and $N_{1}$ meet the surfaces on which $N$ and $N_{1}$ lie in these nets. Conversely the most general transformation $T$ is defined in this way.

From (7) we have by differentiation

$$
\frac{\partial x_{1}}{\partial u}=\frac{\tau}{\theta^{\prime 2}}\left(x^{\prime} \frac{\partial \theta}{\partial u}-\theta^{\prime} \frac{\partial x}{\partial u}\right), \quad \frac{\partial x_{1}}{\partial v}=\frac{\sigma}{\theta^{\prime 2}}\left(x^{\prime} \frac{\partial \theta}{\partial v}-\theta^{\prime} \frac{\partial x}{\partial v}\right),
$$

where we have put

$$
\tau=h \theta-\theta^{\prime}, \quad \sigma=l \theta-\theta^{\prime} .
$$

\footnotetext{
*Annales de Toulouse, ser. 2, vol. 10 (1908), pp. 125-164.
} 
Hence the first fundamental coefficients $E_{1}, F_{1}, G_{1}$ of $N_{1}$ are given by

$$
\begin{aligned}
& E_{1}=\frac{\tau^{2}}{{\theta^{\prime}}^{2}}\left[\sum{x^{\prime}}^{2} \cdot\left(\frac{\partial \theta}{\partial u}\right)^{2}-2 \sum x^{\prime} \frac{\partial x}{\partial u} \cdot \theta^{\prime} \frac{\partial \theta}{\partial u}+{\theta^{\prime}}^{2} E\right], \\
& F_{1}=\frac{\tau \sigma}{{\theta^{\prime}}^{2}}\left[\sum{x^{\prime}}^{2} \cdot \frac{\partial \theta}{\partial u} \frac{\partial \theta}{\partial v}-\sum x^{\prime} \frac{\partial x}{\partial u} \cdot \theta^{\prime} \frac{\partial \theta}{\partial v}-\sum x^{\prime} \frac{\partial x}{\partial v} \cdot \theta^{\prime} \frac{\partial \theta}{\partial u}+{\theta^{\prime}}^{2} F\right], \\
& G_{1}=\frac{\sigma^{2}}{\theta^{\prime 2}}\left[\sum{x^{\prime}}^{2} \cdot\left(\frac{\partial \theta}{\partial v}\right)^{2}-2 \sum x^{\prime} \frac{\partial x}{\partial v} \cdot \theta^{\prime} \frac{\partial \theta}{\partial v}+{\theta^{\prime 2}}^{2}\right] .
\end{aligned}
$$

Suppose now that $N$ admits an applicable net $\bar{N}(\bar{x})$. Since $a$ and $b$ in (5) are functions of the first fundamental coefficients of $N$, and consequently of $\bar{N}$, a net $\bar{N}^{\prime}\left(\bar{x}^{\prime}\right)$ parallel to $\bar{N}$ is defined by the quadratures

$$
\frac{\partial \bar{x}^{\prime}}{\partial u}=h \frac{\partial \bar{x}}{\partial u}, \quad \frac{\partial \bar{x}^{\prime}}{\partial v}=l \frac{\partial \bar{x}}{\partial v} .
$$

It is readily seen that $N^{\prime}$ and $\bar{N}^{\prime}$ are applicable, a result due to Peterson.*

Since $\theta$ and $\theta^{\prime}$ are corresponding solutions of the point equations of $\bar{N}$ and $\bar{N}^{\prime}$, a transform $\bar{N}_{1}$ of $\bar{N}$ is given by

$$
\bar{x}_{1}=\bar{x}-\frac{\theta}{\theta^{\prime}} \bar{x}^{\prime} \text {. }
$$

Ordinarily $N_{1}$ and $\bar{N}_{1}$ are not applicable. We seek the conditions under which they are applicable. From (10) and analogous equations for $\bar{N}_{1}$ we find that for this to be true it is necessary and sufficient that

$$
\begin{aligned}
& \left(\sum{x^{\prime}}^{2}-\sum{\bar{x}^{\prime}}^{2}\right) \frac{\partial \theta}{\partial u}=2\left(\sum x^{\prime} \frac{\partial x}{\partial u}-\sum \bar{x}^{\prime} \cdot \frac{\partial \bar{x}}{\partial u}\right) \theta^{\prime}, \\
& \left(\sum{x^{\prime}}^{2}-\sum \bar{x}^{\prime 2}\right) \frac{\partial \theta}{\partial v}=2\left(\sum x^{\prime} \frac{\partial x}{\partial v}-\sum \bar{x}^{\prime} \frac{\partial \bar{x}}{\partial v}\right) \theta^{\prime},
\end{aligned}
$$

which in consequence of (6) are equivalent to

$$
\theta^{\prime}=\sum{x^{\prime}}^{2}-\sum{\overline{x^{\prime}}}^{2}
$$

to within a negligible constant factor. Koenigs called attention to the fact that when two applicable surfaces are referred to the common conjugate system and consequently have the same point equation, a solution of the latter is given by the difference of the sum of the squares of the coördinates of each surface. Hence $\theta^{\prime}$ as given by (13) is a solution of the point equation of $N^{\prime}$ and $\bar{N}^{\prime}$, and the corresponding solution of the point equation of $N$ is given by the quadrature

(14) $\frac{\partial \theta}{\partial u}=2\left(\sum x^{\prime} \frac{\partial x}{\partial u}-\sum \bar{x}^{\prime} \frac{\partial \bar{x}}{\partial u}\right), \quad \frac{\partial \theta}{\partial v}=2\left(\sum x^{\prime} \frac{\partial x}{\partial v}-\sum \bar{x}^{\prime} \frac{\partial \bar{x}}{\partial v}\right)$.

* Loc. cit. 
Hence we have the

THEOREM. If $N$ and $\bar{N}$ are applicable nets, each net parallel to $N$ determines a pair of applicable nets $N_{1}$ and $\bar{N}_{1}$, which are the respective transforms of $N$ and $\bar{N}$.

\section{Permanent nets}

A permanent net, as defined in the Introduction, is characterized by the property that its curves have the same spherical representation as the asymptotic lines of a surface whose total curvature is of the form

$$
K=-\frac{1}{\rho^{2}}, \quad \rho=\phi(u)+\psi(v),
$$

where $\phi$ and $\psi$ are functions of $u$ and $v$ alone respectively, the parameters referring to the net.* There are three cases to be considered, according as $\rho$ is constant, involves only one parameter, or both parameters. By a suitable choice of the parameters we can reduce the cases to the canonical forms: $\rho=$ const., $\rho=v, \rho=u+v$. We consider these cases in detail, excluding for the present the possibility of the curves in one or both systems on the sphere being the isotropic generators.

$1^{\circ}$. When $\rho=a$, a constant, the coefficients of the linear element of the spherical representation are reducible to the form

$$
\mathcal{E}=1, \quad \mathcal{F}=\cos 2 \omega, \quad \mathcal{G}=1,
$$

the function $\omega$ being a solution of

$$
2 \frac{\partial^{2} \omega}{\partial u \partial v}+\sin 2 \omega=0 . \dagger
$$

If $D, D^{\prime \prime}$ and $D_{k}, D_{k}^{\prime \prime}$ denote the second fundamental coefficients of $N$ and of an applicable net $\bar{N}$, then for any of the three cases

$$
D_{k}=\tanh \phi \cdot D, \quad D_{k}^{\prime \prime}=\operatorname{coth} \phi \cdot D^{\prime \prime},
$$

where $\phi$ is given by

$$
\frac{\partial \phi}{\partial u}=\left\{\begin{array}{c}
12 \\
2
\end{array}\right\}^{\prime} \tanh \phi, \quad \frac{\partial \phi}{\partial v}=\left\{\begin{array}{c}
12 \\
1
\end{array}\right\}^{\prime} \operatorname{coth} \phi,
$$

the Christoffel symbols being formed with respect to the linear element of the spherical representation. $\neq$

For the case $\rho=a$ we have

$$
D_{k}=\frac{D}{k}, \quad D_{k}^{\prime \prime}=k D^{\prime \prime},
$$

\footnotetext{
${ }^{*}$ E., p. 340. A reference of this sort is to the author's Differential Geometry.

$\dagger$ E., p. 190.

$\ddagger$ E., p. 340.
} 
where $k$ is a constant such that when $k=1$ we have the net $N$. From the general formulas connecting the fundamental coefficients of a surface, namely*

$$
\begin{aligned}
\mathcal{E}\left(E G-F^{2}\right)= & G D^{2}, \quad \mathcal{F}\left(E G-F^{2}\right)=-F D D^{\prime \prime}, \\
& \mathcal{G}\left(E G-F^{2}\right)=E D^{\prime 2},
\end{aligned}
$$

we find for the coefficients of the spherical representation of $N$ the expressions

$$
\mathcal{E}_{k}=\frac{1}{k^{2}}, \quad \mathscr{F}_{k}=\cos 2 \omega, \quad \mathcal{G}_{k}=k^{2} .
$$

Incidentally we observe that the pseudospherical surfaces with these spherical representations of their asymptotic lines are in the relation of transformations of Lie. $\dagger$

$2^{\circ}$. When $\rho=v$, equations (34), E., p. 340 become

$$
\left\{\begin{array}{c}
12 \\
1
\end{array}\right\}^{\prime}=-\frac{1}{2 v}, \quad\left\{\begin{array}{c}
12 \\
2
\end{array}\right\}^{\prime}=0,
$$

so that the coefficients of the spherical representation of $N$ are reducible to

$$
\mathcal{E}=\frac{1}{v}, \quad \mathscr{F}=-\frac{\partial \psi}{\partial u}, \quad \mathcal{S}=\frac{\psi}{v},
$$

where $\psi$ is a solution of a partial differential equation of the third order obtained by equating to unity the expression for the Gaussian curvature of the linear element of the spherical representation.

In this case the integral of (18) is of the form

$$
\tanh \phi=\sqrt{1+k v},
$$

where $k$ is a constant. Hence we have

$$
\begin{gathered}
D_{k}=\sqrt{1+k v} D, \quad D_{k}^{\prime \prime}=\frac{D^{\prime \prime}}{\sqrt{1+k v}}, \\
\mathcal{E}_{k}=\frac{1}{v}+k, \quad \mathscr{F}_{k}=-\frac{\partial \psi}{\partial u}, \quad \mathcal{G}_{k}=\frac{\psi}{v+k v^{2}}, \\
\rho_{k}=\frac{v}{1+k v},
\end{gathered}
$$

where $-1 / \rho_{k}^{2}$ is the total curvature of the surface whose asymptotic lines have the spherical representation with the coefficients (25).

$3^{\circ}$. When $\rho=u+v$, we have

${ }^{*}$ E., p. 200.

$$
\left\{\begin{array}{c}
12 \\
1
\end{array}\right\}^{\prime}=\frac{-1}{2(u+v)}, \quad\left\{\begin{array}{c}
12 \\
2
\end{array}\right\}^{\prime}=\frac{-1}{2(u+v)},
$$

† E., p. 289. 
from which it follows that we may take

$$
\mathcal{E}=\frac{\partial \psi}{\partial u}(u+v)^{-1}, \quad \mathcal{F}=-\frac{\partial^{2} \psi}{\partial u \partial v}, \quad \mathcal{S}=\frac{\partial \psi}{\partial v}(u+v)^{-1},
$$

the function $\psi$ being determined by the Gauss equation of the sphere as in the former case.

The integral of the corresponding equations (18) is

so that

$$
\tanh \phi=\sqrt{\frac{1+k v}{1-k u}} \equiv \lambda,
$$

and

$$
D_{k}=\lambda D, \quad D_{k}^{\prime \prime}=\frac{1}{\lambda} D^{\prime \prime},
$$

$$
\mathcal{E}_{k}=\frac{\lambda^{2}}{u+v} \frac{\partial \psi}{\partial u}, \quad \mathscr{F}_{k}=-\frac{\partial^{2} \psi}{\partial u \partial v}, \quad \mathcal{G}_{k}=\frac{1}{\lambda^{2}(u+v)} \frac{\partial \psi}{\partial v},
$$

with the result

$$
\rho_{k}=\frac{u+v}{(1-k u)(1+k v)},
$$

$k$ being a constant such that when $k=0$ we have the original net $N$.

\section{Transformations $\Omega$}

Let $X, Y, Z$ be the direction-cosines of the normal to a net $N$ and $W$ the fourth tangential coördinate so that

$$
X x+Y y+Z z=W .
$$

The net $N$ is said to have equal tangential invariants when the equation which these coördinates satisfy has equal invariants. We suppose that $N$ is such a net, the tangential equation being

$$
\frac{\partial^{2} \theta}{\partial u \partial v}+\frac{1}{2} \frac{\partial \log \rho}{\partial v} \frac{\partial \theta}{\partial v}+\frac{1}{2} \frac{\partial \log \rho}{\partial u} \frac{\partial \theta}{\partial v}+\sqrt{\mathcal{E S}} \cos 2 \omega \cdot \theta=0,
$$

and the linear element of the spherical representation

$$
d \sigma^{2}=\mathcal{E} d u^{2}+2 \sqrt{\mathcal{E S}} \cos 2 \omega d u d v+\mathcal{G} d v^{2} .
$$

If $X^{\prime}, Y^{\prime}, Z^{\prime}$ and $X^{\prime \prime}, Y^{\prime \prime}, Z^{\prime \prime}$ denote the direction-cosines of the bisectors of the angles between the parametric lines on the sphere, we find with the aid of the Gauss equations* for the sphere the following:

*E., p. 160. 


$$
\begin{array}{lll}
\frac{\partial X}{\partial u}=\sqrt{\mathcal{E}}\left(\sin \omega X^{\prime}+\cos \omega X^{\prime \prime}\right), & \frac{\partial X}{\partial v}=\sqrt{\mathcal{G}}\left(-\sin \omega X^{\prime}+\cos \omega X^{\prime \prime}\right), \\
\frac{\partial X^{\prime}}{\partial u}=-A X^{\prime \prime}-\sqrt{\mathcal{E}} \sin \omega X, & \frac{\partial X^{\prime}}{\partial v}=B X^{\prime \prime}+\sqrt{\mathcal{S}} \sin \omega X, \\
\frac{\partial X^{\prime \prime}}{\partial u}=A X^{\prime}-\sqrt{\mathcal{E}} \cos \omega X, & \frac{\partial X^{\prime \prime}}{\partial v}=-B X^{\prime}-\sqrt{\mathcal{S}} \cos \omega X,
\end{array}
$$

where

$$
A=\frac{\partial \omega}{\partial u}-\sqrt{\frac{\mathcal{E}}{\mathcal{G}}} \sin 2 \omega \frac{\partial \log \sqrt{\rho}}{\partial v}, \quad B=\frac{\partial \omega}{\partial v}-\sqrt{\frac{\mathcal{G}}{\mathcal{E}}} \sin 2 \omega \frac{\partial \log \sqrt{\rho}}{\partial u} .
$$

The curves on the sphere represent the asymptotic lines on a surface $\Sigma$ whose total curvature is $-1 / \rho^{2}$.

From the theory of tangential coördinates we know that if $W^{\prime}$ is any other solution of equation (32), the functions $X, Y, Z, W^{\prime}$ are the tangential coördinates of a net $N^{\prime}$ parallel to $N$. This net leads to transformations $T$ of $N$ further determined by solutions of the point equation of $N$. In particular one of these transforms, $N_{1}$, is defined in terms of its tangential coördinates $X_{1}, Y_{1}, Z_{1}, W_{1}$, which are given by

$$
\begin{aligned}
& \frac{\partial}{\partial u}\left(\sqrt{\rho \rho_{1}} W^{\prime} X_{1}\right)=\rho\left(W^{\prime} \frac{\partial X}{\partial u}-X \frac{\partial W^{\prime}}{\partial u}\right), \\
& \frac{\partial}{\partial v}\left(\sqrt{\rho \rho_{1}} W^{\prime} X_{1}\right)=-\rho\left(W^{\prime} \frac{\partial X}{\partial v}-X \frac{\partial W^{\prime}}{\partial v}\right),
\end{aligned}
$$

and similar equations when $X_{1}$ and $X$ are replaced by $Y_{1}, Y ; Z_{1}, Z$; and $W_{1}, W$ respectively, the function $\rho_{1}$ being a function of $u$ and $v$ such that $-1 / \rho_{1}^{2}$ is the total curvature of the surface $\Sigma_{1}$ with the same spherical representation of its asymptotic lines as the curves of $N_{1}{ }^{*}$ We have said that in this case the nets $N$ and $N_{1}$ are in the relation of a transformation $\Omega$.

It is convenient to express $X_{1}$ in the form

$$
X_{1}=\cos \sigma X+\sin \sigma\left(\sin \alpha X^{\prime}-\cos \alpha X^{\prime \prime}\right),
$$

where as thus defined $\sigma$ is the angle between the tangent planes to $N$ and $N_{1}$.

Suppose now that $N$ is a permanent net. We have shown $\dagger$ that $N_{1}$ also is a permanent net, provided that $W^{\prime}, \sigma$, and $\alpha$ satisfy the completely integrable system of equations

$$
\frac{\partial \sigma}{\partial u}=\cot \frac{\sigma}{2} \frac{\partial \log \rho}{\partial u}, \quad \frac{\partial \sigma}{\partial v}=-\tan \frac{\sigma}{2} \frac{\partial \log \rho}{\partial v},
$$

* These Transactions, l. c., n. 119.

† Rendicontidi Palermo, loc. cit. 


$$
\begin{aligned}
\frac{\partial \log W^{\prime}}{\partial u} & =\sqrt{\mathcal{E}} \cot \frac{\sigma}{2} \cos (\alpha+\omega)-\frac{\partial \log \rho}{\partial u} \frac{1}{1-\cos \sigma}, \\
\frac{\partial \log W^{\prime}}{\partial v} & =-\sqrt{\mathcal{S}} \tan \frac{\sigma}{2} \cos (\alpha-\omega)-\frac{\partial \log \rho}{\partial v}-\frac{1}{1+\cos \sigma}, \\
\frac{\partial \alpha}{\partial u} & =A-\sqrt{\mathcal{E}} \cot \frac{\sigma}{2} \sin (\alpha+\omega), \\
\frac{\partial \alpha}{\partial v} & =-B+\sqrt{\mathfrak{S}} \tan \frac{\sigma}{2} \sin (\alpha-\omega) .
\end{aligned}
$$

In this case the parallel net of the transformation is of a special kind, and will be called a special permanent net.

\section{Solutions of problem $A$ when $\rho=a$}

A permanent net $N$ and a parallel net $N^{\prime}$ admit continuous arrays of deforms $N^{(k)}$ and $N^{(k)^{\prime}}$ such that for the same value of $k$ these nets are parallel. By means of $N^{\prime}$ and the function

$$
\theta_{k}^{\prime}=\sum{x^{\prime}}^{2}-\sum x_{k}^{\prime 2}
$$

we obtain a transform $N_{1}^{(k)}$ of $N$, and at the same time by means of $\theta_{k}^{\prime}$ and $N^{(k)^{\prime}}$ a transform $\left(N^{(k)}\right)_{1}$ of $N^{(k)}$ such that $N_{1}^{(k)}$ and $\left(N^{(k)}\right)_{1}$ are applicable. Ordinarily $N_{1}^{(k)}$ varies with $k$. If it does not, it is a permanent net admitting the family of applicable nets $\left(N^{(k)}\right)_{1}$. We shall show that if we take any permanent net $N$ and for $N^{\prime}$ one of the special permanent nets as defined in $\S 3$, the transforms $N_{1}^{(k)}$ coincide for all values of $k$ and thus we have solutions of Problem $A$.

We denote by $\sigma_{k}, \alpha_{k}, w_{k}$ solutions of equations (38) defining a special net $N^{(k)^{\prime}}$ parallel to $N^{(k)}$. From (15) and (21) we see that $\omega$ is independent of $k$. Hence for $\rho=a$ we have, since $\rho_{k}=a$ also,

$$
\begin{aligned}
& \frac{\partial \sigma_{k}}{\partial u}=0, \quad \frac{\partial \sigma_{k}}{\partial v}=0, \\
& \frac{\partial \log w_{k}}{\partial u}=\frac{1}{k} \cot \frac{\sigma_{k}}{2} \cos \left(\alpha_{k}+\omega\right), \\
& \frac{\partial \log }{\partial v} \frac{w_{k}}{\partial}=-\frac{1}{k} \tan \frac{\sigma_{k}}{2} \cos \left(\alpha_{k}-\omega\right), \\
& \frac{\partial \alpha_{k}}{\partial u}-\frac{\partial \omega}{\partial u}+\frac{1}{k} \cot \frac{\sigma_{k}}{2} \sin \left(\alpha_{k}+\omega\right)=0, \\
& \frac{\partial \alpha_{k}}{\partial v}+\frac{\partial \omega}{\partial v}-k \tan \frac{\sigma_{k}}{2} \sin \left(\alpha_{k}-\omega\right)=0 \text {. }
\end{aligned}
$$


The second fundamental coefficients $D_{k}$ and $D_{k}^{\prime \prime}$ of the net whose tangential coördinates are $X, Y, Z, w_{k}$ are given by the general formulas*

$$
\begin{aligned}
& D_{k}=-\left(\frac{\partial^{2} w_{k}}{\partial u^{2}}-\left\{\begin{array}{c}
11 \\
1
\end{array}\right\}^{\prime} \frac{\partial w_{k}}{\partial u}-\left\{\begin{array}{c}
11 \\
2
\end{array}\right\}^{\prime} \frac{\partial w_{k}}{\partial v}+\mathcal{E}_{k} w_{k}\right), \\
& D_{k}^{\prime \prime}=-\left(\frac{\partial^{2} w_{k}}{\partial v^{2}}-\left\{\begin{array}{c}
22 \\
1
\end{array}\right\}^{\prime} \frac{\partial w_{k}}{\partial u}-\left\{\begin{array}{l}
22 \\
2
\end{array}\right\}^{\prime} \frac{\partial w_{k}}{\partial v}+\mathcal{S}_{k} w_{k}\right),
\end{aligned}
$$

where the Christoffel symbols are formed wich respect to the linear element of the spherical representation, its coefficients having the values (21). Making use of $(40)$ we find

$$
\begin{aligned}
& D_{k}=\frac{w_{k}}{k \sin \frac{\sigma_{k}}{2}}\left[\frac{\cos \left(\alpha_{k}-\omega\right)}{\sin 2 \omega} \frac{2 \frac{\partial \omega}{\partial u}}{\cos \frac{\sigma_{k}}{2}}-\frac{1}{k \sin \frac{\sigma_{k}}{2}}\right], \\
& D_{k}^{\prime \prime}=-\frac{k w_{k}}{\cos \frac{\sigma_{k}}{2}}\left[\frac{\cos \left(\alpha_{k}+\omega\right)}{\sin 2 \omega} \frac{2 \frac{\partial \omega}{\partial v}}{\sin \frac{\sigma_{k}}{2}}+\frac{k}{\cos \frac{\sigma_{k}}{2}}\right] .
\end{aligned}
$$

The values for the net $N^{\prime}$ are given by (41') when $k=1$. It is readily found that the conditions (19) are satisfied, if we take

$$
\cot \frac{\sigma_{k}}{2}=k \cot \frac{\sigma}{2}, \quad \alpha_{k}=\alpha, \quad w_{k}=\frac{k w}{\sin ^{2} \frac{\sigma}{2}+k^{2} \cos ^{2} \frac{\sigma}{2}} .
$$

Moreover, these values are consistent with (40).

It remains for us to calculate the expression for $\theta_{k}^{\prime}$ as defined by (39). The cartesian coördinates $x, y, z$, of a surface, whose tangential coördinates are $X, Y, Z, W$, are given by expressions of the form

$$
x=W X+\frac{1}{\mathcal{E S}-\mathcal{F}^{2}}\left[\mathcal{S} \frac{\partial W}{\partial u} \frac{\partial X}{\partial u}-\mathcal{F}\left(\frac{\partial W}{\partial u} \frac{\partial X}{\partial v}+\frac{\partial W}{\partial v} \frac{\partial X}{\partial u}\right)+\mathcal{E} \frac{\partial W}{\partial v} \frac{\partial X}{\partial v}\right] .
$$

From this it is readily found that

$$
\sum x^{2}=W^{2}+\frac{1}{\mathcal{E S}-\mathcal{F}^{2}}\left[\mathcal{S}\left(\frac{\partial W}{\partial u}\right)^{2}-2 \mathcal{F} \frac{\partial W}{\partial u} \frac{\partial W}{\partial v}+\mathcal{E}\left(\frac{\partial W}{\partial v}\right)^{2}\right]
$$

Applying this to the case where $w_{k}$ and $w$ are given by (40) and (42), we find

\footnotetext{
*E., p. 164.
}

$$
\theta_{k}^{\prime}=\frac{w^{2} \sin 2 \alpha}{\sin 2 \omega} \cdot \frac{\sin ^{2} \frac{\sigma}{2}+k^{2} \cos \sigma-k^{4} \cos ^{2} \frac{\sigma}{2}}{\left(\sin ^{2} \frac{\sigma}{2}+k^{2} \cos ^{2} \frac{\sigma}{2}\right)^{2}}
$$


Hence $\theta_{k}^{\prime}$ varies with $k$ only in a constant factor, so that from (6) and (7) it follows that the transform $N_{1}$ does not vary with $k$.

\section{Solutions of Problem $A$ When $\rho=v$ AND When $\rho=u+v$}

We consider next the case when equations (22) and (23) hold. The integral of the first two of equations (38) is

$$
\tan ^{2} \frac{\sigma_{k}}{2}=\frac{b(1+k v)}{v-b},
$$

where $b$ is a constant, and the other equations become

$$
\begin{aligned}
& \frac{\partial \log w_{k}}{\partial u}=\sqrt{\frac{v-b}{v b}} \cos \left(\alpha_{k}+\omega\right), \\
& \frac{\partial \log w_{k}}{\partial v}=-\sqrt{\psi} \sqrt{\frac{b}{v^{2}-v b}} \cos \left(\alpha_{k}-\omega\right)-\frac{b k+1}{2(v-b)(1+k v)},
\end{aligned}
$$

$$
\begin{aligned}
& \frac{\partial \alpha_{k}}{\partial u}-\frac{\partial \omega}{\partial u}+\frac{1}{2 v \sqrt{\psi}} \sin 2 \omega+\sqrt{\frac{v-b}{v b}} \sin \left(\alpha_{k}+\omega\right)=0, \\
& \frac{\partial \alpha_{k}}{\partial v}+\frac{\partial \omega}{\partial v}-\sqrt{\psi} \sqrt{\frac{b}{v^{2}-b v}} \sin \left(\alpha_{k}-\omega\right)=0 .
\end{aligned}
$$

From (41) we have in this case

$$
\begin{aligned}
& -\frac{D_{k}}{w_{k}} \frac{1}{1+b k}=\frac{1}{b}+\frac{1-2 v^{2} \frac{\partial^{2} \psi}{\partial u^{2}}}{2 v\left[\psi-v^{2}\left(\frac{\partial \psi}{\partial u}\right)^{2}\right]}\left(\frac{1}{2(v-b)}+\frac{\sqrt{\psi v} \cos \left(\alpha_{k}-\omega\right)}{\sqrt{b v(v-b)}}\right) \\
& -\frac{D_{k}^{\prime \prime \prime}}{w_{k}} \frac{1}{1+b k}=\frac{3}{2} \cos \left(\alpha_{k}-\omega\right) \sqrt{\frac{b}{v}} \frac{\sqrt{\psi}}{(v-b)^{\frac{3}{2}}}+\frac{1}{4(v-b)^{2}}\left(4-\frac{b}{v}\right) \\
& +\sqrt{\frac{b v}{v-b}} \frac{1}{\left[\psi-v^{2}\left(\frac{\partial \psi}{\partial u}\right)^{2}\right]}\left(v \psi \frac{\partial^{2} \psi}{\partial u \partial v}+\psi \frac{\partial \psi}{\partial u}-\frac{v}{2} \frac{\partial \psi}{\partial u} \frac{\partial \psi}{\partial v}\right) \cos \left(\alpha_{k}+\omega\right) \\
& \quad+\frac{1}{4(v-b)} \frac{1}{\frac{\psi}{v^{2}}-\left(\frac{\partial \psi}{\partial u}\right)^{2}}\left(\frac{1}{v^{2}} \frac{\partial \psi}{\partial v}-2 \frac{\partial \psi}{\partial u} \frac{\partial^{2} \psi}{\partial u \partial v}-\frac{2 \psi}{v}\right)
\end{aligned}
$$

A comparison of these results and (24) shows that these conditions are met, if we treat $b$ as independent of $k$ and take

$$
\alpha_{k}=\alpha, \quad w_{k}=\frac{\sqrt{1+k v}}{1+k b} w .
$$


The net $N^{\prime}$ corresponds to the value $k=0$, and $w$ for $N^{\prime}$ is given by (45) for this value of $k$. Moreover, the latter are consistent with (45) and (46).

Making use of (43), we find

$$
\theta_{k}^{\prime}=-\frac{w^{2} k v}{\sin 2 \omega(b k+1)}\left(\sin 2 \alpha+\frac{1}{\sqrt{\psi}} \sqrt{\frac{b}{v^{2}-b v}} \sin (\alpha+\omega)\right)
$$

Hence $\theta_{k} / \theta_{k}^{\prime}$ is independent of $k$, and all the transforms $N_{1}$ coincide.

For the case where the coefficients of the linear element of the spherical representation have the form (30) the equations analogous to (44) and (45) are

$$
\tan ^{2} \frac{\sigma_{k}}{2}=\lambda^{2} \frac{u+b}{v-b},
$$

and

$$
\begin{aligned}
& \frac{\partial \log w_{k}}{\partial u}=\frac{\sqrt{\frac{\partial \psi}{\partial u}}}{\sqrt{u+v}} \sqrt{\frac{v-b}{u+b}} \cos \left(\alpha_{k}+\omega\right)-\frac{b k+1}{2(u+b)(1-k u)}, \\
& \frac{\partial \log w_{k}}{\partial v}=-\frac{\sqrt{\frac{\partial \psi}{\partial v}}}{\sqrt{u+v}} \sqrt{\frac{u+b}{v-b}} \cos \left(\alpha_{k}-\omega\right)-\frac{b k+1}{2(v-b)(1+k v)}, \\
& \frac{\partial \alpha_{k}}{\partial u}-\frac{\partial \omega}{\partial u}+\sqrt{\frac{\partial \psi}{\partial u}} \frac{\sin 2 \omega}{2(u+v)} / \sqrt{\frac{\partial \psi}{\partial v}} \\
& +\sqrt{\frac{\partial \psi}{\partial u}} \frac{\sqrt{v-b}}{\sqrt{(u+v)(u+b)}} \sin \left(\alpha_{k}+\omega\right)=0, \\
& \frac{\partial \alpha_{k}}{\partial v}+\frac{\partial \omega}{\partial v}-\sqrt{\frac{\partial \psi}{\partial v}} \frac{\sin 2 \omega}{2(u+v)} / \sqrt{\frac{\partial \psi}{\partial u}} \\
& -\sqrt{\frac{\partial \psi}{\partial v}} \frac{\sqrt{u+b}}{\sqrt{(u+v)(v-b)}} \sin \left(\alpha_{k}-\omega\right)=0 .
\end{aligned}
$$

Proceeding as in the former cases with the evaluation of $D_{k}$ and $D_{k}^{\prime \prime}$, we find

$$
\frac{D_{k}(1-k u)}{w_{k}(1+b k)}=A, \quad \frac{D_{k}^{\prime \prime}(1+k v)}{w_{k}(1+b k)}=B,
$$

where $A$ and $B$ represent certain complicated expressions not involving $k$, on the assumption that $b$ is independent of $k$ and that $\alpha_{k}=\alpha$. It is seen that these values are consistent with (29), if we take

$$
w_{k}=\frac{w}{1+b k} \sqrt{(1+k v)(1-k u)},
$$


which expression satisfies (49) on the assumption that $w \equiv w_{0}$. Now

$$
\begin{aligned}
\theta_{k}^{\prime}=-\frac{k w^{2}(u \dashv \cdot v)}{\sin 2 \omega(1+b k)} \sqrt{\frac{\partial \psi}{\partial u} \cdot \frac{\partial \psi}{\partial v}}\left(\sin 2 \alpha \sqrt{\frac{\partial \psi}{\partial u} \frac{\partial \psi}{\partial v}}\right. & \\
& -\frac{1}{\sqrt{u+v}} \sqrt{\frac{\partial \psi}{\partial v}} \sqrt{\frac{v-b}{u+b}} \sin (\alpha-\omega) \\
& \left.+\frac{1}{\sqrt{u+v}} \sqrt{\frac{\partial \psi}{\partial u}} \sqrt{\frac{u+b}{v-b}} \sin (\alpha+\omega)\right) .
\end{aligned}
$$

As in the two preceding cases $\theta_{k} / \theta_{k}^{\prime}$ is independent of $k$.

In view of these results we have the

Theorem. Given a family of applicable permanent nets $N_{i}$, of any of the three types, and a family of special permanent nets $N_{i}^{\prime}$ parallel to the former; each net $N_{i}$ is transformable by means of its parallel and the function determined by any other net $N_{j}^{\prime}$ into a net $N_{1}$ which is independent of the choice of $N_{j}^{\prime}$; the family of transforms thus obtained are deforms of one another.

6. Permanent nets with the curves in one family Represented on the GaUsS SPHERE BY ISOTROPIC GENERATORS

Drach* determined the equations of all nets of this kind. He showed that the coefficients of the spherical representation of such a net are reducible to the form

$$
\mathcal{E}=0, \quad \mathcal{F}=\frac{2}{(1+u v)^{2}}, \quad \mathcal{G}=\frac{2 V^{\prime}}{V v(1+u v)}+V_{1},
$$

where $V$ and $V_{1}$ are functions of $v$ alone; also that the tangential coördinates $X, Y, Z, W$, of such a net are expressible in the form

$$
\begin{gathered}
X=\frac{a_{1}}{1+u v}+b_{1}, \quad Y=\frac{a_{2}}{1+u v}+b_{2}, \quad Z=\frac{a_{3}}{1+u v}+b_{3}, \\
W \sqrt{V}=2 \frac{v \phi(u)+u \psi(v)}{1+u v}-\phi^{\prime}-\psi^{\prime},
\end{gathered}
$$

where $\phi$ and $\psi$ are arbitrary functions of $u$ and $v$ alone and the $a$ 's and $b$ 's are functions of $v$ alone subject to the conditions:

$$
\begin{gathered}
\sum_{i=1}^{3} a_{i}^{2}=0, \quad \sum_{i=1}^{3} a_{i}^{\prime 2}=\frac{4}{v^{2}}, \quad \sum_{i=1}^{3} a_{i}^{\prime 2}=\frac{4}{v^{2}}\left(V_{1}-\frac{5}{4} \frac{V^{\prime 2}}{V^{2}}+\frac{2 V^{\prime}}{v V}+\frac{V^{\prime \prime}}{V}\right), \\
b_{i}=\left(\frac{V^{\prime} v}{4} \frac{1}{V}-\frac{1}{2}\right) a_{i}+\frac{v}{2} a_{i}^{\prime} \quad(i=1,2,3),
\end{gathered}
$$

the primes indicating differentiation with respect to the argument.

\footnotetext{
* Loc. cit.
} 
If $X^{\prime}, Y^{\prime}, Z^{\prime}$ denote the direction-cosines of the tangent to the curves $u=$ const. on the sphere, and $X^{\prime \prime}, Y^{\prime \prime}, Z^{\prime \prime}$, the direction-cosines of the tangents to the orthogonal trajectories of these curves, we have

$$
\frac{\partial X}{\partial u}=\frac{\mathcal{F}}{\sqrt{\mathcal{S}}}\left(X^{\prime}+i X^{\prime \prime}\right), \quad \frac{\partial X}{\partial v}=\sqrt{\mathfrak{S}} X^{\prime} .
$$

Proceeding as in the determination of the formulas (34), we obtain

$$
\begin{array}{ll}
\frac{\partial X^{\prime}}{\partial u}=-i \frac{V^{\prime}}{2 V} \frac{\mathcal{F}}{\mathcal{G}} X^{\prime \prime}-\frac{\mathcal{F}}{\sqrt{\mathcal{G}}} X, & \frac{\partial X^{\prime}}{\partial v}=i\left\{\begin{array}{l}
22 \\
1
\end{array}\right\}^{\prime} \frac{\mathcal{F}}{\mathcal{G}} X^{\prime \prime}-\sqrt{\mathcal{S}} X, \\
\frac{\partial X^{\prime \prime}}{\partial u}=i \frac{V^{\prime}}{2 V} \frac{\mathcal{F}}{\mathcal{G}} X^{\prime}-i \frac{\mathcal{F}}{\sqrt{\mathcal{G}}} X, & \frac{\partial X^{\prime \prime}}{\partial v}=-i\left\{\begin{array}{l}
22 \\
1
\end{array}\right\}^{\prime} \frac{\mathcal{F}}{\mathcal{G}} X^{\prime},
\end{array}
$$

where

$$
\left\{{ }_{1}^{22}\right\}^{\prime}=\frac{1}{2 \mathcal{F}^{\prime}} \frac{\partial \mathcal{G}}{\partial v}+\frac{\mathcal{G}}{2 \mathcal{F}^{2}} \frac{\partial \mathcal{G}}{\partial u}-\frac{\mathcal{G}}{\mathcal{F}^{2}} \frac{\partial \mathscr{F}}{\partial v} .
$$

The tangential coördinates satisfy the equation

$$
\frac{\partial^{2} \theta}{\partial u \partial v}=-\frac{V^{\prime}}{2 V} \frac{\partial \theta}{\partial u}-\mathcal{F} \theta,
$$

which evidently has equal invariants. Hence the curves on the sphere represent the asymptotic lines on a surface $\Sigma$. We are interested in finding the surfaces $\Sigma_{1}$ such that $\Sigma$ and $\Sigma_{1}$ are the focal surfaces of a $W$-congruence. We assume that the direction-cosines of the normal to $\Sigma_{1}$ are given by equations of the form (37), where $\alpha$ and $\sigma$ are to be determined. We recall $*$ that these direction-cosines must satisfy equations of the form

$$
\begin{aligned}
& \frac{\partial}{\partial u}\left(\sqrt{V \rho_{1}} w X_{1}\right)=-V w^{2} \frac{\partial}{\partial u}\left(\frac{X}{w}\right), \\
& \frac{\partial}{\partial v}\left(\sqrt{V \rho_{1}} w X_{1}\right)=V w^{2} \frac{\partial}{\partial v}\left(\frac{X}{w}\right),
\end{aligned}
$$

where $w$ is a solution of (55) and $\rho_{1}$ is the function in the tangential equation for $\Sigma_{1}$ analogous to (32). When the expressions (37) are substituted in (56), and it is noted that similar results must hold also for the $Y$ 's and $Z$ 's, we are led to the following equations of condition:

*E., p. 419. 


$$
\begin{gathered}
\frac{\partial \sigma}{\partial u}=\frac{i \mathcal{F}}{\sqrt{\mathcal{S}}} e^{i \alpha}\left(1+\cos \sigma \sqrt{\frac{V}{\rho_{1}}}\right)-\sqrt{\frac{V}{\rho_{1}}} \sin \sigma \frac{\partial \log w}{\partial u} \\
\frac{\partial \sigma}{\partial v}=-\sqrt{\mathcal{G}} \sin \alpha\left(1-\cos \sigma \sqrt{\frac{V}{\rho_{1}}}\right)+\sqrt{\frac{V}{\rho_{1}}} \sin \sigma \frac{\partial \log w}{\partial v} \\
\frac{\partial}{\partial u} \log \sqrt{V \rho_{1}}+\frac{\partial \log w}{\partial u}\left(1-\cos \sigma \sqrt{\frac{V}{\rho_{1}}}\right)-i \frac{\mathcal{F}}{\sqrt{\mathcal{S}}} e^{i \alpha} \sin \sigma \sqrt{\frac{V}{\rho_{1}}}=0, \\
\frac{\partial}{\partial v} \log \sqrt{V \rho_{1}}+\frac{\partial \log w}{\partial v}\left(1+\cos \sigma \sqrt{\frac{V}{\rho_{1}}}\right)-\sqrt{\mathcal{S}} \sin \alpha \sin \sigma \sqrt{\frac{V}{\rho_{1}}}=0 \\
\sin \sigma\left(\frac{\partial \alpha}{\partial u}-i \frac{V^{\prime}}{2 V} \frac{\mathcal{F}}{\mathcal{S}}\right)+\frac{\mathcal{F}}{\sqrt{\mathcal{G}}} e^{i \alpha}\left(\cos \sigma+\sqrt{\frac{V}{\rho_{1}}}\right)=0 \\
\sin \sigma\left(\frac{\partial \alpha}{\partial v}+i\left\{{ }_{1}^{22}\right\}^{\prime} \frac{\mathcal{F}}{\mathcal{G}}\right)+\sqrt{\mathcal{S}} \cos \alpha\left(\cos \sigma-\sqrt{\frac{V}{\rho_{1}}}\right)=0 .
\end{gathered}
$$

By means of these equations the first derivatives of $X_{1}$ are reducible to

$$
\begin{aligned}
& \frac{\partial X_{1}}{\partial u}=-\left(\cos \sigma \frac{\partial \log w}{\partial u}+i \frac{\mathcal{F}}{\sqrt{\mathcal{G}}} e^{i a} \sin \sigma\right) \sqrt{\frac{V}{\rho_{1}}}\left[\cos \sigma X+\left(\sin \alpha X^{\prime}\right.\right. \\
& \left.\left.\quad-\cos \alpha X^{\prime \prime}\right) \sin \sigma\right]-\sqrt{\frac{V}{\rho_{1}}} \frac{\mathcal{F}}{\sqrt{\mathcal{G}}}\left(X^{\prime}+i X^{\prime \prime}\right)+X \sqrt{\frac{V}{\rho_{1}}} \frac{\partial \log w}{\partial u}, \\
& \frac{\partial X_{1}}{\partial v}=\left(\cos \sigma \frac{\partial \log w}{\partial v}-\sqrt{G} \sin \alpha \sin \sigma\right) \sqrt{\frac{V}{\rho_{1}}}[\cos \sigma X \\
& \left.+\left(\sin \alpha X^{\prime}-\cos \alpha X^{\prime \prime}\right) \sin \sigma\right]+\sqrt{\frac{V}{\rho_{1}}} \sqrt{\mathcal{S}} X^{\prime}-X \sqrt{\frac{V}{\rho_{1}}} \frac{\partial \log w}{\partial v} .
\end{aligned}
$$

From these equations we find the following expressions for the coefficients $\mathcal{E}_{1}$ of the linear element of the spherical representation of $\Sigma_{1}$ :

$$
\mathcal{E}_{1}=\frac{V}{\rho_{1}}\left[\left(\frac{\partial \log w}{\partial u}\right)^{2}-\left(\cos \sigma \frac{\partial \log w}{\partial u}+i \frac{\mathcal{F}}{\sqrt{\mathfrak{S}}} e^{i \alpha} \sin \sigma\right)\right] .
$$

We are interested particularly in the case where the curves $v=$ const. on the sphere are minimal, that is when $\mathcal{E}_{1}=0$. From the above we have

$$
\frac{\partial \log w}{\partial u}=i \frac{\mathcal{F}}{\sqrt{\mathcal{G}}} e^{i a} \frac{\sin \sigma}{1-\cos \sigma} .
$$

Since the tangential equation is to be of the form (55), $\rho_{1}$ is a function of $v$ alone. From (58) and the third of (57) we find that $\rho_{1}=V . *$ Hence equa-

* It should be remarked that the possible change of signs in (58) consistent with $\mathcal{E}_{1}=0$ is merely equivalent to replacing $\sigma$ by $\sigma+\pi$, and consequently introduces nothing different from the above. 
tions (57) reduce to

$$
\begin{gathered}
\frac{\partial \sigma}{\partial u}=0, \quad \frac{\partial \sigma}{\partial v}=-\tan \frac{\sigma}{2} \cdot \frac{V^{\prime}}{V}, \\
\frac{\partial \log w}{\partial u}=i \frac{\mathcal{F}}{\sqrt{\mathcal{S}}} e^{i a} \cot \frac{\sigma}{2}, \\
\frac{\partial \log w}{\partial v}=\sqrt{\mathcal{G}} \sin \alpha \tan \frac{\sigma}{2}-\frac{1}{1+\cos \sigma} \frac{V^{\prime}}{V}, \\
\frac{\partial \alpha}{\partial u}-\frac{i}{2} \frac{V^{\prime}}{V} \frac{\mathcal{F}}{\mathcal{G}}+\frac{\mathcal{F}}{\sqrt{\mathcal{S}}} e^{i a} \cot \frac{\sigma}{2}=0, \\
\frac{\partial \alpha}{\partial v}+i\left\{{ }_{1}^{22}\right\}^{\prime} \frac{\mathcal{F}}{\mathcal{G}}-\sqrt{\mathcal{S}} \cos \alpha \tan \frac{\sigma}{2}=0 .
\end{gathered}
$$

From these we get

$$
\begin{aligned}
\frac{\partial}{\partial u} \log w e^{i a} & =-\frac{V^{\prime}}{2 V} \frac{\mathcal{F}}{\mathcal{G}}=\frac{\partial}{\partial u} \log \sqrt{\mathcal{S}}, \\
\frac{\partial}{\partial v} \log w e^{i a} & =\left\{{ }_{1}^{22}\right\}^{\prime} \frac{\mathcal{F}}{\mathcal{S}}+i \sqrt{\mathcal{S}} \tan \frac{\sigma}{2} e^{-i a} .
\end{aligned}
$$

Moreover, from the first two of (59) we find

$$
\tan \frac{\sigma}{2}=\sqrt{\frac{a}{V-a}}
$$

where $a$ is a constant. The function $w$ is of the form (51). Substituting this value in the third of (59), we get

$$
\sqrt{V} i \frac{w e^{i a}}{\sqrt{\mathcal{S}}} \cot \frac{\sigma}{2}=(1+u v) v \phi^{\prime}+\psi-v^{2} \phi-\frac{\phi^{\prime \prime}}{2}(1+u v)^{2} .
$$

From (62) and the first of (61) we see that the left-hand member of this equation is necessarily a function of $v$ alone. In order that the expression in the right-hand member be a function of $v$ alone, we must have

$$
\phi=b u^{2}+2 c u+d,
$$

where $b, c$, and $d$ are constants. Then, if we put $\psi-d v^{2}+2 c v-b=\bar{V}$, equation (63) reduces to

and we have also

$$
\sqrt{V} i w e^{i a} \cot \frac{\sigma}{2}=\sqrt{\mathcal{S}} \bar{V},
$$

$$
\sqrt{V} w=\frac{2 u \bar{V}}{1+u v}-\bar{V}^{\prime}
$$


In order that these values may satisfy the second of equations (61), it is necessary that $V$ be a constant, in which case $\sigma$ also is constant. From these two equations we have

$$
e^{-i a}=\frac{2 i u \cot \frac{\sigma}{2}}{(1+u v) \sqrt{V_{1}}}+V_{2}, \quad V_{2}=-\frac{\bar{V}^{\prime}}{\overline{\bar{V}}} \frac{i}{\sqrt{V_{1}}} \cot \frac{\sigma}{2} .
$$

All of the conditions (59) and (60) will be satisfied, if this value satisfies the last of (61). This gives an equation of Riccati for the determination of $V_{2}$, whence $\psi$ is found by a quadrature. Hence we have the

THEOREM. When the asymptotic lines of a surface $\Sigma$ have the spherical representation with the coefficients (50), the determination of other surfaces $\Sigma_{1}$ of the same kind, such that $\Sigma$ and $\Sigma_{1}$ are the focal surfaces of a W-congruence, requires the integration of a Riccati equation and quadratures; and this is possible only when the coefficient $\mathcal{G}$ is a function of $v$ alone.

\section{Solutions of PROBlem $A$ FOR PERMANent NETS FOR WHich $\mathcal{E}=0$}

We apply the results of the preceding section and obtain a solution of Problem $A$ for the nets whose spherical representation has coefficients of the form

$$
\mathcal{E}=0, \quad \mathcal{F}=\frac{2}{(1+u v)^{2}}, \quad \mathcal{G}=V_{1} .
$$

Since in this case we have

$$
\left\{\begin{array}{l}
12 \\
1
\end{array}\right\}^{\prime}=\left\{\begin{array}{c}
12 \\
2
\end{array}\right\}^{\prime}=0,
$$

equations (17) assume the form (19), and in place of (21) we have

$$
\mathcal{E}_{k}=0, \quad \mathscr{F}_{k}=\mathscr{F}, \quad \mathcal{S}_{k}=\mathscr{G} k^{2} .
$$

Proceeding as in $\S 4$, we find that in this case equations (42) hold also and the function $\theta_{k}^{\prime}$ is given by

$$
\theta_{k}^{\prime}=w_{k}^{2} e^{2 i a} \frac{k^{2}-1}{\sin ^{2} \frac{\sigma}{2}+k^{2} \cos ^{2} \frac{\sigma}{2}} .
$$

Hence $\theta_{k} / \theta_{k}^{\prime}$ is independent of $k$, and we have a solution of Problem $A$.

Drach* solved the problem of the deformation of permanent nets with the coefficients (50). We recall his results. If $R$ is a general solution of the Riccati equation

where

$$
R^{\prime}+\frac{1}{2} R^{2}+\frac{1}{2} \Omega=0,
$$

$$
\Omega=\frac{T V}{1+k V}+\frac{3}{4} \frac{V^{\prime 2}}{(1+k V)^{2} V^{2}},
$$

* Loc. cit.

Trans. Am. Math. Soc. 13 
$k$ being a parameter and $R$ and $T$ functions of $v$ alone, and if we put

$$
\begin{gathered}
\rho=e^{\int R d v}, \quad \sigma=\frac{1}{2} \int \frac{d v}{\rho}, \\
A_{1}=\rho\left(1-\sigma^{2}\right), \quad A_{2}=i \rho\left(1+\sigma^{2}\right), \quad A_{3}=2 \rho \sigma,
\end{gathered}
$$

then the functions $X, Y, Z$, given by the expressions

$$
-2 \frac{A_{i} u}{1+u v}+A_{i}^{\prime}-A_{i} \frac{\partial}{\partial v} \log \sqrt{\frac{1}{V}+k},
$$

as $i$ takes the values $1,2,3$ respectively, are the direction-cosines and $W_{k}$, given by

$$
\dot{W}_{k}=\sqrt{\frac{1}{V}+k}\left(2 \frac{v \phi(u)+u \psi(v)}{1+u v}-\phi^{\prime}(u)-\psi^{\prime}(v)\right),
$$

the fourth tangential coördinate of a net with the coefficients (50). Moreover if in (64) we put

$$
\psi=\Psi V / \sqrt{1+k V},
$$

where $\Psi$ is any solution of the equation

$$
\Psi^{\prime \prime \prime}+\Omega \Psi^{\prime}+\frac{1}{2} \Omega^{\prime} \Psi=\frac{\Phi V}{\sqrt{1+k V}},
$$

$\Phi$ being an arbitrary function of $v$, then all the surfaces obtained by varying $k$ are applicable.

The coefficients of the spherical representation of these surfaces are of the form

$$
\begin{gathered}
\mathcal{E}_{k}=0, \quad \mathcal{F}_{k}=\frac{2}{(1+u v)^{2}} \\
\mathcal{G}_{k}=\frac{V}{1+k V}\left[\frac{2 u}{1+u v}\left(\frac{1}{V}\right)^{\prime}+\left(\frac{1}{V}\right)^{\prime \prime}+T\right] .
\end{gathered}
$$

We consider in particular the surfaces for which $\psi=0$. Making use of the general formula (43) and denoting by $x_{k}^{\prime}, y_{k}^{\prime}, z_{k}^{\prime}$ the cartesian coördinates of the surface with these tangential coördinates, we find

$$
\begin{array}{r}
\theta_{k}^{\prime}=\sum x_{k}^{\prime 2}-\sum x_{0}^{\prime 2}=k\left[\frac{2 \phi}{(1+u v)^{2}}\left((1+u v) v \phi^{\prime}-v^{2} \varphi-\frac{1}{2}(1+u v)^{2} \phi^{\prime \prime}\right)\right. \\
\left.+\left(2 \frac{v \phi}{1+u v}-\phi^{\prime}\right)^{2}\right] .
\end{array}
$$

From the form of this expression it follows that the ratio $\theta_{k}^{\prime} / \theta_{k}$ is independent of $k$, and consequently by means of these functions $\theta_{k}$ and $\theta_{k}^{\prime}$ we get a transformation of any of the surfaces with the tangential coördinate (64), which is a solution of Problem $A$. 
9. WhEN THE CURVES IN BOTH SYSTEMS ARE REPRESENTED ON THE SPHERE BY GENERATORS

There remains for consideration the case when the curves in both systems of a net are represented on the sphere by imaginary generators, that is when the surface on which the net lies is minimal. In this case we say that the net $N$ is minimal. The coördinates of $N$ and $\bar{N}$ are necessarily of the form*

$$
x=U+V, \quad \bar{x}=e^{i k} U+e^{-i k} V,
$$

where $k$ is a constant, and the three functions $U$ of $u$ alone and the three functions $V$ of $v$ alone are subject to the conditions

$$
\sum U^{\prime 2}=0, \quad \sum V^{\prime 2}=0 .
$$

Parallel nets $N^{\prime}$ and $\bar{N}^{\prime}$ are necessarily given by

$$
x^{\prime}=h U+l V, \quad \bar{x}^{\prime}=e^{i k} h U+e^{-i k} l V,
$$

where $h$ and $l$ are any unequal constants. Now

$$
\begin{aligned}
& \theta_{k}^{\prime}=h^{2}\left(1-e^{2 i k}\right) \sum U^{2}+l^{2}\left(1-e^{-2 i k}\right) \sum V^{2}, \\
& \theta_{k}=h\left(1-e^{2 i k}\right) \sum U^{2}+l\left(1-e^{-2 i k}\right) \sum V^{2} .
\end{aligned}
$$

In order that $\theta^{\prime} / \theta$ be independent of $k$, we must have either $\Sigma U^{2}=0$, or $\Sigma V^{2}=0$. From the Weierstrass form $\dagger$ of the functions $U$ and $V$ it follows that only when the $U$ 's are constant can they satisfy simultaneously the conditions $\Sigma U^{2}=0, \sum U^{\prime 2}=0$. Hence in all transformations of this sort $N_{1}$ varies with $k$. Making use of formulas (10), we find that in this case the nets $N_{1}$ are not minimal.

Princeton University

* E., p. 327.

† E., p. 262. 